\title{
POTENCIAL DE DISPARIDAD
}

\author{
MISMATCH NEGATIVITY
}

Loreto Carrasco $\mathrm{M}^{1}$, Elizabeth Pavez $\mathrm{A}^{2}$ y Paul H. Délano $\mathrm{R}^{3}$.

\begin{abstract}
RESUMEN
日 potencial de disparidad o mismatch negativity $(M M N)$ corresponde a la respuesta eléctrica extraída del electroencefalograma que se produce frente a diversos cambios de las características del estímulo acústico. Se obtiene presentando al sujeto una secuencia de estímulos repetitivos de características acústicas similares (estímulo estándar) alternado en forma aleatoria con estímulos acústicos discrepantes que difieren del primero en alguno de sus atributos (estímulo discrepante). originaría en la corteza auditiva primaria y se ha logrado registrar desde el nacimiento. No requiere la atención del sujeto, permitiendo evaluar en forma objetiva la discriminación de tonos y fonemas. En clínica se ha utilizado en evaluación del procesamiento auditivo central, pacientes con dislexia o trastorno específico del lenguaje, autismo, individuos con implante coclear e incluso en pacientes en coma. En el presente artículo se revisan las principales características, origen anatómico y utilidad clínica del MMN.

Palabras clave: Mismatch negativity, potencial de disparidad, procesamiento auditivo central, corteza auditiva, memoria auditiva, potenciales relacionados a evento.
\end{abstract}

\section{ABSTRACT}

The mismatch negativity (MMN) is a specific component of the auditory eventrelated brain potentials. It is elicited by an infrequent, physically deviant sound (deviantstimulus) occurring in a sequence of homogeneous repetitive sounds (standard-stimulus). MMN is probably generated in the primary auditory cortex and it has been successfully recorded in newborns. The MMN can be elicited even in the absence of attention and it can be used as an objective method to assess tone and phoneme discrimination. Some clinical applications of MMN are: evaluation of central auditory processing, patients with dyslexia or language specific disorders, autism, cochlear implant - users and even in prognosis of coma. In this article the main characteristics, origin and clinical applications of the MMN are reviewed.

Key words: Mismatch negOativity, central auditory processing, auditory cortex, auditory memory, event-related brain potentials

1 Médico, Magíster en Ciencias Médicas, mención Morfología.

2 Tecnólogo Médico, Magíster en Biofísica Médica. Escuela Tecnología Médica. Facultad de Medicina, Universidad de Chile.

3 Médico, PhD. Servicio Otorrinolaringología, Hospital Clínico de la Universidad de Chile. 


\section{INTRODUCCIÓN}

En 1939, se describió por primera vez que un estímulo acústico puede alterar el electroencefalograma de un ser humano despierto' ${ }^{1}$. Desde entonces se han estudiado diversos tipos de potenciales auditivos de origen central, como: (i) potenciales de tronco encefálico, que se caracterizan por tener latencias menores a $10 \mathrm{~ms}$ desde el inicio del estímulo, (ii) potenciales de latencia media, con latencias entre 1080 ms y (iii) potenciales de latencia tardía (N1, P1, N2, $\mathrm{P} 3$, y potencial de disparidad o mismatch negativity) con latencias sobre los $80 \mathrm{~ms}^{2,3}$.

Los potenciales auditivos de tronco encefálico fueron descritos por Jewett y Williston en $1971^{4}$. Estos se generan por la descarga sincrónica de grupos de neuronas que conforman la vía auditiva central. Debido a que son reproducibles y confiables han sido ampliamente utilizados en clínica para evaluar umbrales auditivos sensoriales y realizar topodiagnóstico de una pérdida auditiva ${ }^{2-4}$.

Los potenciales auditivos corticales de latencia tardía pueden ser clasificados en sensoriales y cognitivos, siendo los primeros directamente dependientes de las características acústicas del estímulo utilizado, mientras que los últimos se modifican con eventos cognitivos. Dentro de los potenciales sensoriales encontramos a las ondas $\mathrm{P} 1, \mathrm{~N} 1$ y $\mathrm{P} 2$, mientras que en los potenciales cognitivos encontramos a P3, también denominado p300 por su latencia cercana a $300 \mathrm{~ms}^{2}$. El potencial de disparidad o mismatch negativity (como se conoce ampliamente en la literatura), es un potencial de latencia tardía que se obtiene con diseños similares a los utilizados para obtener p300, pero a diferencia de éste, el potencial de disparidad no depende de la atención del sujeto.

Otro tipo de potencial cortical que evalúa las respuestas de la corteza auditiva a diferentes frecuencias son los potenciales de estado estable, descritos por Galambos y cols. en $1981^{5}$. Estos permiten obtener una medición audiométrica objetiva $^{6}$. La gran variabilidad que se produce entre sujetos al obtener potenciales auditivos corticales ha dificultado su implementación en la práctica clínica diaria. En este artículo revisaremos las características del mismatch negativity (MMN), discutiendo su origen anatómico, desarrollo y sus posibles aplicaciones clínicas.

\section{CARACTERÍSTICAS DEL MISMATCH NEGATIVITY}

El potencial de disparidad o mismatch negativity, descrito por Risto Näätänen y colaboradores en $1978^{7}$, corresponde a la respuesta eléctrica extraída del electroencefalograma que se produce frente a diversos cambios de las características del estímulo acústico ${ }^{8}$.

El MMN se obtiene presentando al sujeto una secuencia de estímulos repetitivos de características acústicas similares (estímulo estándar) alternado en forma aleatoria con estímulos acústicos discrepantes que difieren del primero en alguno de sus atributos (frecuencia, intensidad, duración, etc.). Habitualmente, el estímulo estándar es presentado en el $80 \%-90 \%$ de los ensayos (frecuente), mientras que el estímulo discrepante se presenta en el 10\%-20\% de los ensayos (infrecuente), aunque también se han utilizado otras proporciones $^{8,9}$. Ambos estímulos, tanto el estándar como el discrepante, producen un potencial evocado sensorial (P1, N1 y P2), pero sólo el estímulo discrepante produce un desplazamiento negativo registrado con electrodos ubicados específicamente en la región fronto-central y central (Fz y Cz). Para obtener el MMN se restan los potenciales promediados del estímulo estándar y del discrepante (Figura 1). Se ha encontrado un correlato electrofisiológico del MMN obtenido en seres humanos en diversas especies de mamíferos como: monos $^{10}$, gatos ${ }^{11}$, cobayos $^{12}$, ratas $^{13}$ y ratones ${ }^{14}$.

La latencia del pico del MMN se produce entre 100 a 250 milisegundos desde el inicio del estímulo discrepante ${ }^{15}$. A mayor diferencia de frecuencias entre el estímulo estándar y discrepante, la amplitud del MMN es mayor y su latencia disminuye ${ }^{16}$. Además del MMN que se obtiene por diferencia de frecuencias, el MMN puede ser provocado por estímulos auditivos que difieren en intensidad, duración, frecuencia de presentación y localización espacial17-21. Se ha obtenido MMN con pares de fonemas, utilizando a uno como estímulo estándar y un segundo como estímulo discrepante. Además, se ha obtenido MMN con sonidos naturales o con pasajes musicales 22,23 .

El MMN puede obtenerse sin la atención del sujeto, incluso en pacientes en estado de coma ${ }^{24}$. Se ha demostrado además que el MMN se 


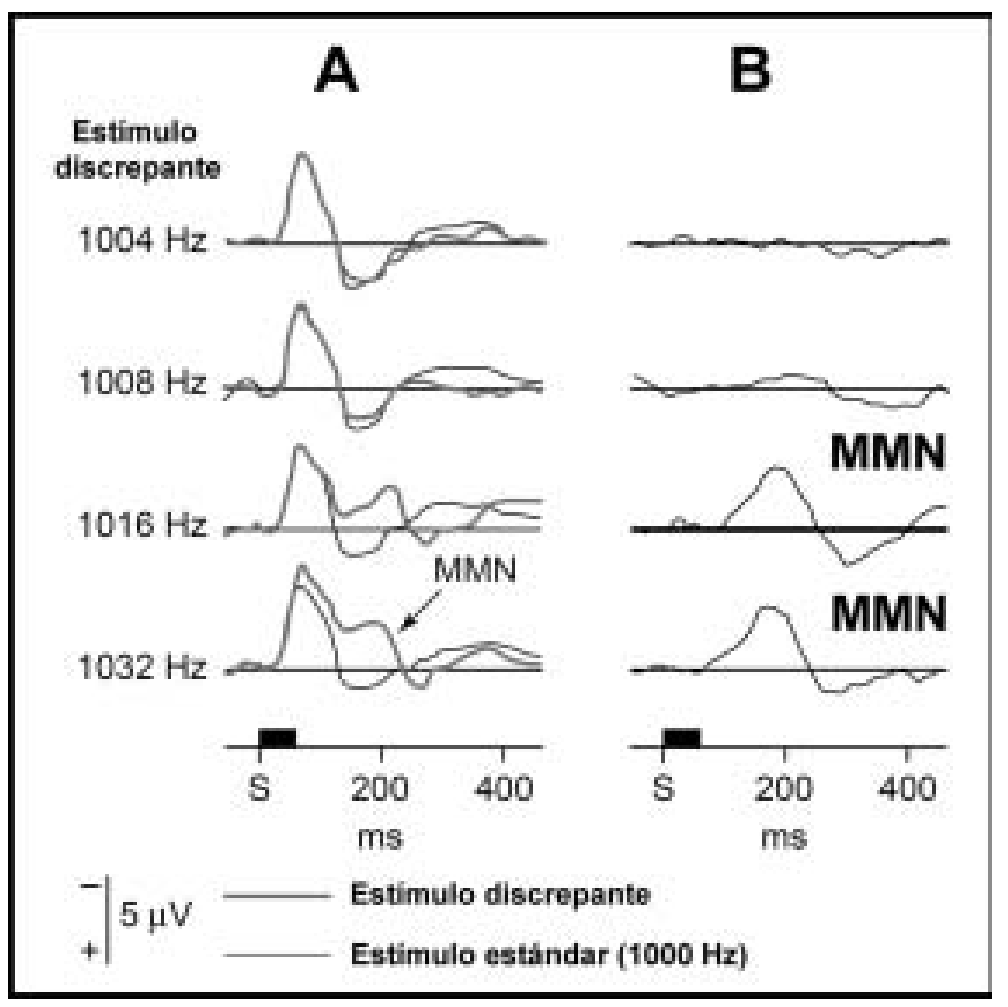

Figura 1. Mismatch negativity (MMN) obtenido por diferencias de frecuencias entre los estímulos estándar y discrepante. A. Se muestran los trazados de potenciales evocados por estímulos estándar (línea fina) y por estímulos discrepantes (línea gruesa). Los estímulos estándar $(1000 \mathrm{~Hz}$ ) fueron presentados en el 80\% de los ensayos, mientras que los discrepantes $(1004-1032 \mathrm{~Hz}$ ) fueron presentados en el 20\% de los ensayos. La flecha indica la presencia de MMN para el estímulo discrepante de $1032 \mathrm{~Hz}$.

B. Se muestran los trazados que se obtienen al restar las ondas promediadas de los estímulos estándar y discrepante. Se observa que para los estímulos de 1016 y $1032 \mathrm{~Hz}$ se obtiene MMN. Durante la obtención de estos potenciales, los sujetos se encontraban leyendo un libro (Figura modificada de Näätänen $2001^{9}$ ).

correlaciona con el desempeño de sujetos en tareas de discriminación de frecuencias, así los sujetos que son buenos en tareas de discriminación evocan un MMN de gran amplitud, mientras que aquellos que tienen un bajo porcentaje de discriminación no evocan MMN²5. Esto implica que el MMN puede ser utilizado como una medida objetiva de la discriminación auditiva.

\section{MMN y memoria auditiva}

En sujetos jóvenes y sanos se puede obtener un MMN con estímulos discrepantes presentados hasta 5 a 10 segundos después del estímulo estándar ${ }^{26,27}$. Esto muestra que en sujetos sanos la corteza auditiva mantiene actividad eléctrica relacionada al estímulo estándar por cerca de 10 segundos, lo que se cree puede corresponder a la duración de la memoria auditiva de trabajo. Además se ha demostrado que este período va disminuyendo con la edad ${ }^{28}$. Utilizando un tiempo entre estímulos auditivos de 4,5 segundos, los sujetos de edad avanzada generan un MMN de menor amplitud que los sujetos jóvenes. Esto sugiere que con la edad va disminuyendo la duración de la memoria sensorial de tipo auditiva ${ }^{29}$. Así el MMN podría ser utilizado como una medida objetiva de la duración de la memoria auditiva de corto plazo.

\section{Origen del MMN}

Utilizando un modelo de dipolos se localizó al generador del MMN en el plano supratemporal de las cortezas auditivas derecha e izquierda ${ }^{30}$. Otro estudio en que se utilizó un modelo de densidad de corrientes también situó como fuente del MMN a las cortezas auditivas ${ }^{31}$. 
Estudios realizados con magneto-encefalografía (donde se registra el equivalente magnético del MMN que se abrevia mMMN) también sugieren que el origen del MMN se produce en o cerca de la corteza auditiva primaria $^{32}$. Se ha registrado el MMN en pacientes con epilepsia que fueron sometidos a mapeos electrofisiológicos de la corteza cerebral durante neurocirugías. En estos pacientes se corroboró que el origen del MMN sería la corteza auditiva contralateral al oído estimulado. Estudios realizados en gatos con electrodos intracorticales demuestran la presencia de MMN en la corteza auditiva primaria y en sus áreas circundantes ${ }^{11}$. Recientemente se ha confirmado, con estudios realizados en seres humanos con resonancia magnética funcional, que el origen del MMN reside en la corteza auditiva primaria33. No obstante, algunos estudios desarrollados en animales han sugerido que el tálamo auditivo podría ser el origen del $\mathrm{MMN}^{34,35}$ lo que ha sido apoyado por estudios que muestran que lesiones talámicas alteran el MMN ${ }^{36}$. Sin embargo, se necesitan más trabajos para confirmar que el tálamo está involucrado en la génesis del MMN.

\section{Desarrollo del sistema auditivo central y $M M N$}

El MMN se puede obtener en recién nacidos (RN) despiertos 0 dormidos con características similares a las observadas en adultos ${ }^{37-39}$. Una diferencia del MMN registrado en RN comparado con el obtenido en adultos es la duración de la memoria auditiva. En RN no se obtiene MMN con intervalos entre estímulos mayores a 1 segundo, mientras que en adultos el MMN puede ser obtenido incluso con intervalos de 10 segundos $^{27,40}$. Se ha demostrado además que en algunos RN se evoca MMN con diferentes vocales ${ }^{41}$ y más aún se ha demostrado que la discriminación de fonemas se puede entrenar durante el sueño en RN, lo que se manifiesta por la aparición de MMN después de presentar pares de fonemas durante una noche ${ }^{38,40}$. Recientemente, se ha demostrado utilizando magneto-encefalografía con electrodos colocados sobre el abdomen de mujeres embarazadas, que el mMMN es posible obtenerlo en fetos de 33 semanas de gestación ${ }^{42}$.

\section{Aplicación clínica del MMN}

Se ha propuesto el uso del MMN en el estudio de diversas patologías audiológicas y neurológicas, como procesamiento auditivo central, implante coclear, trastornos del lenguaje, evaluación de pacientes en coma y otras (Tabla 1).

\section{Tabla 1. Aplicaciones clínicas del MMN}

- Evaluación objetiva del procesamiento auditivo central

- Evaluación de disfunciones fonológicas y auditivas en dislexia y trastorno específico del lenguaje

- Autismo

- Implante coclear

- Pronóstico en pacientes con traumatismo encéfalocraneano y coma

- Otras: Demencia tipo Alzheimer, enfermedad de Parkinson, esquizofrenia

\section{Procesamiento auditivo central}

La evaluación del procesamiento auditivo central (PAC) busca conocer la eficiencia y eficacia con que el sistema auditivo central maneja la información auditiva que recibimos desde el medio externo. Este proceso comprende mecanismos y funciones responsables de la localización y lateralización del sonido, discriminación auditiva, reconocimiento de patrones auditivos y aspectos temporales de la audición como resolución, enmascaramiento, integración y orden, además de evaluación del rendimiento auditivo en condiciones de una señal acústica competitiva o pobre ${ }^{43,44}$. Las alteraciones del PAC pueden repercutir en alteraciones de la voz, del habla, el lenguaje y la lectoescritura, así como en el aprendizaje y déficit atencionales ${ }^{45,46}$.

Para evaluar el PAC existen pruebas psicoacústicas y electrofisiológicas. Las pruebas de comportamiento incluyen pruebas de detección de palabras con ruido de fondo, prueba de dígitos dicóticos, palabras filtradas, fusión binaural, detección de intervalos de silencio y secuencias temporales $^{46,47}$. Si bien estas pruebas permiten evaluar las alteraciones del PAC, el MMN permitiría evaluar el PAC en forma objetiva, y sin necesitar de la colaboración ni atención del sujeto.

El MMN ha sido utilizado para evaluar disfunciones fonológicas y auditivas en pacientes con dislexia y con trastornos específicos del lenguaje $^{48}$. De un total de 26 trabajos publicados en 
MMN y trastornos del lenguaje al año 2007, se muestra que el principal hallazgo en los niños con trastornos del lenguaje y dislexia fue la disminución de amplitud del $\mathrm{MMN}^{48}$. Sin embargo aún existe gran variabilidad en los protocolos utilizados en estos trabajos de investigación, lo que dificulta la implementación del MMN en la evaluación de este grupo de pacientes.

\begin{abstract}
Autismo
El MMN ha sido estudiado en pacientes con síndrome de Asperger, enfermedad que cae dentro del espectro de trastornos autistas. Estos pacientes presentan un aumento de la amplitud del MMN para los estímulos discrepantes obtenidos con intervalos de silencio o con estímulos de corta duración. Además se observa un acortamiento de la latencia del MMN para los cambios de frecuen$\mathrm{cia}^{49-51}$. Estos resultados sugieren que estos pacientes presentan mayor capacidad auditiva para detectar cambios acústicos.
\end{abstract}

\section{Implante coclear}

Kraus y cols. ${ }^{52}$ en 1993 estudiaron las características del MMN en 8 adultos con sordera post-lingual con implante coclear. Para obtener el MMN utilizaron estímulos sintéticos de los fonemas / da/ y /ta, que los pacientes discriminaban sin problemas. En este trabajo se encontró que la magnitud y morfología del MMN fue similar en el grupo de implantados que en sujetos normales.

El MMN también ha sido obtenido en pacientes implantados por sordera bilateral poslingual utilizando tonos puros (estímulo estándar $1 \mathrm{kHz}$ y discrepante 2 ó $1,5 \mathrm{kHz}$ ). En este trabajo se observó que la forma de la respuesta (tanto latencia como amplitud) fue similar en los pacientes implantados y en controles. Además no presentaron diferencias de la amplitud del MMN registrado ipsilateral 0 contralateral al implante coclear, sugiriendo que la estimulación eléctrica del nervio auditivo activa ambos hemisferios en sujetos con hipoacusia profunda bilateral ${ }^{53}$.

También se ha estudiado el MMN en niños con sordera profunda bilateral prelingual con implante coclear (tiempo transcurrido tras el implante fue de por lo menos 45 meses), comparados con niños normales, utilizando tonos de 500 y $2500 \mathrm{~Hz}$, sin encontrar diferencias significativas en la magnitud del $\mathrm{MMN}^{54}$.

Estos trabajos muestran que el MMN en pacientes implantados es similar al que se obtiene en controles normales. Sin embargo, éstos han sido realizados en pacientes implantados con buen desempeño en tareas psico-acústicas ${ }^{52-54}$. Sin embargo otros estudios han encontrado que sólo en algunos de los pacientes con implantes cocleares se puede obtener un MMN. Esto se debería a las variaciones individuales de los pacientes implantados, lo que se correlaciona con el desempeño que tienen en tareas de discriminación de fonemas ${ }^{55,56}$. Esta dificultad en la obtención del MMN en algunos individuos ha limitado su utilidad clínica en pacientes con implante coclear.

\section{Traumatismo encéfalocraneano y coma}

Otro posible uso clínico del MMN es el pronóstico de pacientes en coma ${ }^{57-59}$. Kane y cols., observaron que la presencia de MMN anticiparía la recuperación del estado de coma ${ }^{60,61}$. Se propone a MMN como uno de los indicadores más precoces de la recuperación del coma ${ }^{60}$. Posteriormente se realizó un estudio prospectivo, longitudinal en pacientes durante un coma traumático. La presencia de MMN predijo la recuperación del coma y la mejoría con respecto a la escala de Glasgow (sensibilidad de $89,7 \%$ y especificidad de $100 \%$ ). El mejor indicador como seguimiento de un estado comatoso por 90 días fue la latencia del MMN¹.

Se ha estudiado las características del MMN en una serie de patologías neurológicas como: enfermedad de Alzheimer, enfermedad de Parkinson e incluso en la esquizofrenia, pero aún no existen evidencias claras de su utilidad en clínica ${ }^{62-64}$.

\section{CONCLUSIONES}

El MMN es un potencial auditivo tardío, de origen central que está presente desde el nacimiento y que permite evaluar en forma objetiva la discriminación de tonos y fonemas. El MMN será, probablemente, de gran utilidad en la evaluación clínica de pacientes con patología audiológica y neurológica. Sin embargo, debido a que es un potencial técnicamente difícil de obtener y que 
presenta gran variabilidad individual, su utilidad en clínica es aún limitada, siendo actualmente utilizado fundamentalmente en investigación.

\section{Agradecimientos}

Agradecemos al Dr. Diego Elgueda por sus valiosos comentarios en la preparación del presente manuscrito.

\section{BIBLIOGRAFÍA}

1. Davis PA. Effects of Acoustic stimuli on the Waking Human Brain. J Neurophysiol 1939; 2: 494-9.

2. Cone-Wesson B, Wunderlich J. Auditory evoked potentials from the cortex: audiology applications. Ourr Opin Otolaryngol Head Neck Surg 2003; 11: 372-7.

3. De Cosmo G, Aceto P, Clemente A, Congedo E. Auditory evoked potentials. Minerva Anestesiol 2004; 70: 293-7.

4. Jewett DL, Williston JS. Auditory-evoked far fields averaged from the scalp of humans. Brain 1971; 94: 681-96.

5. Galambos R, Makelg S, Talmachoff PJ. A 40-Hz auditory potential recorded from the human scalp. Proc Natl Acad Sci U.S.A. 1981; 78(4): 2643-7.

6. Cabello P, Caro L. Audiometría de Estado Estable. Rev Aorrinolaringol Cir Cabeza Cuello 2007; 67: 162-6.

7. Naatanen R, Gaillard aWK, Mantysalo S. Early selective-attention effect on evoked potential reinterpreted. Acta Psychol 1978; 42: 313-29.

8. Naatanen R, Paavilainen P, Rinne T, Alho K. The mismatch negativity (MMN) in basic research of central auditory processing: A review. Cin Neurophysiol 2007; 118(12): 2544-90.

9. Naatanen R, Tervaniemi M, Sussman E, Paavilainen P, WINKLER I. Primitive intelligence in the auditory cortex. Trends in Neurosci 2001; 24(5): 283-8.

10. Javitt DC, Schroeder CE, Steinschneider M, Arezzo JC, Vaughan HG JR. Demonstration of mismatch negativity in the monkey. Bectroencephalogr Cin Neurophysiol 1992; 83(1): 87-90.

11. CSEPE V. On the origin and development of the mismatch negativity. Ear Hear 1995; 16: 91-104.

12. King C, McGee T, Rubel EW, Nicol T, Kraus N. Acoustic features and acoustic changes are represented by different central pathways. Hear Res 1995; 85(1-2): 45-52.

13. Ruusuvirta T, Penttonen M, Korhonen T. Auditory cortical event-related potentials to pitch deviances in rats. Neurosci Lett 1998; 248(1): 45-8.

14. Umbricht D, Vyssotki D, Latanov A, Nitsch R, Lipp HP. Deviance-related electrophysiological activity in mice: is there mismatch negativity in mice? Cin Neurophysiol 2005; 116(2): 353-63.

15. Lang AH, Eerola 0, Korpilahti P, Holopainen I, Salo S, Aaltonen 0. Practical issues in the clinical application of mismatch negativity. Ear Hear 1995; 16(1): 118-30.

16. Natanen R. The mismatch negativity: a powerful tool for cognitive neuroscience. Ear Hear 1995; 16(1): 6-18.

17. Naatanen R, Teder W, Alho K, Lavikainen J. Auditory attention and selective input modulation: a topographical ERP study. Neuroreport 1992; 3(6): 493-6.

18. Schroger E, Wolff C. Mismatch response of the human brain to changes in sound location. Neuroreport 1996; 7: 3005-9.

19. JaCobsen T, Schroger E. Measuring duration mismatch negativity. Cin Neurophysiol 2003; 114: 1133-43.

20. Jacobsen T, Horenkamp T, Schroger E. Preattentive memory-based comparison of sound intensity. Audiol Neurootol 2003; 8: 338-46.

21. Jacobsen T, Schroger E, Horenkamp T, Winkler I. Mismatch negativity to pitch change: Varied stimulus proportions in controlling effects of neural refractoriness on human auditory event-related potentials. Neurosci Lett 2003; 344: 79-82.

22. Tervaniemi M, Brattico E. From sounds to music. Towards understanding the neurocognition of musical sound perception. $J$ Conscious Stud 2004; 11: 9-27.

23. Natanen R, Lehtokoski A, Lennes M et al. Language-specific phoneme representations revealed by electric and magnetic brain responses. Nature 1997; 385: 432-4.

24. Fischer C, Morlet D, Bouchet P, Luaute J, Jourdan $C$, SaloRd F. Mismatch negativity and late auditory evoked potentials in comatose patients. Cin Neurophysiol 1999; 110: 1601-10.

25. Lang HA, Nyrke T, Ek M, Aaltonen 0, Raimo I, NaAtanen R. Pitch discrimination performance 
and auditory event-related potentials. En: Brunia CHM, Gaillard AWK, Kok A, Mulder G, Verbaten MN, eds. Psychophysiological brain research: Tilburg, the Netherlands: Tilburg University Press; 1990; 1: 294-8.

26. Mantysalo S, naatanen R. The duration of a neuronal trace of an auditory stimulus as indicated by event-related potentials. Biol Psychol 1987; 24: 183-95.

27. Bottcher-Gandor C, Ullsperger P. Mismatch negativity in event-related potentials to auditory stimuli as a function of varying interstimulus interval. Psychophysiology 1992; 29: 546-50.

28. JaAskelainen IP, Hautamaki $M$, Naatanen $R$, ILMONIEMI RJ. Temporal span of auditory sensory memory and mismatch negativity: revisited. Neuroreport 1999; 10: 1305-8.

29. Pekkonen E, Rinne T, Reinikainen K, Kujala T, Alho K, Naatanen R. Aging effects on auditory processing: an event-related potential study. Exp Aging Res 1996; 22: 171-84.

30. Scherg M, Vausar J, Picton TW. A source analysis of the late human auditory evoked potentials. J Cogn Neurosci 1989; 1: 336-55.

31. Giard MH, Perrin F, Pernier J, Bouchet P. Brain generators implicated in processing of auditory stimulus deviance: a topographic event-related potential study. Psychophysiology 1990; 27: 627-40.

32. Hari R, Hamalainen $M$, Ilmoniemi $R$ et al. Responses of the primary auditory cortex to pitch changes in a sequence of tone pips: neuromagnetic recordings in man. Neurosci Lett 1984; 50: 127-32.

33. Molnolm S, Martinez A, Ritter W, Javitt DC, Foxe JJ. The neural circuitry of pre-attentive auditory change-detection: an fMRI study of pitch and duration mismatch negativity generators. Cereb Cortex 2005; 15: 545-51.

34. Csépe V, Karmos G, Molnár M. Subcortical evoked potential correlates of early information processing: mismatch negativity in cats. En: Basar $\mathrm{E}$, Bullock TH, eds. Springer series in brain dynamics. Berlin: Springer Verlag 1989; 2: 278-89.

35. Kraus N, McGee T, Littman T, Nicol T, King C. Nonprimary auditory thalamic representation of acoustic change. J Neurophysiol 1994; 72(3): 1270-7.
36. Makela JP, Salmelin R, Kotila M et al. Modification of neuromagnetic cortical signals by thalamic infarctions. Bectroencephalogr Cin Neurophysiol 1998; 106(5): 433-43.

37. Alho K, Sainio K, Sajaniemi N, Reinikainen K, Natanen R. Event-related potential of human newborns to pitch change of an acoustic stimulus. $\quad$ Eectroencephalogr Cin Neurophysiol 1990; 77(2): 151-5.

38. Cheour M, Ceponiene R, Leppanen P et al. The auditory sensory memory trace decays rapidly in newborns. Scand J Psychol 2002; 43: 33-9.

39. Cheour M, Kushnerenko E, Ceponiene R, Fellman V, NaAtanen R. Electric brain responses obtained from newborn infants to changes in duration in complex harmonic tones. Dev Neurophychol 2002; 22: 471-9.

40. Cheour M, Martynova 0, Naatanen R et al. Speech sounds learned by sleeping newborns. Nature 2002; 415: 599-600.

41. Cheour-Luthanen M, Alho K, Kujala $T$ et al. Mismatch negativity indicates vowel discrimination in newborns. Hear Res 1995; 82(1): 53-8.

42. Draganova R, Eswaran $\mathrm{H}$, Murphy P, Huotilainen M, Lowerey C, Preissl H. Sound frequency change detection in fetuses and newborns, a magnetoencephalographic study. Neurolmage 2005; 28: 354-61.

43. American Speech-Language Hearing Association. Central auditory processing: Current status of research and implications for clinical practice. Am J Audiol 1996; 5(2): 41-54.

44. American Speech-Language-Hearing Association. Central auditory processing disorders - the role of the audiologist (Position statement). 2005; Disponible en: http:// www.asha.org/members/deskref-journals/ deskref/default.

45. KATZ J. Handbook of clinical audiology. 5ㄹ Edición. En: Williams \& Wilkins eds. 1994; 510-24.

46. Cañete OS. Desorden del procesamiento auditivo central. Rev Otorrinolaringol Or Cabeza Quello 2006; 66: 263-73.

47. Fuente A, McPherson B. Auditory processing tests for spanish-speaking adults: An initial study. Int J Audiol 2006; 45: 645-59.

48. BISHOP DV. Using mismatch negativity to study central auditory processing in developmental 
language and literacy impairments: where are we, and where should we be going? Psycholl Bull 2007; 133(4): 651-72.

49. Kujala T, Aho E, Lepisto T et al. Atypical pattern of discriminating sound features in adults with Asperger syndrome reflected by the mismatch negativity. Biol Psychol 2007; 75: 109-14.

50. Dunn W, Myles B.S, OrR S. Sensory processing issues associated with Asperger syndrome: a preliminary investigation. Am J Occup Ther 2002; 56: 97-102.

51. Bonnel A, Mottron L, Peretz I, Trudel M, Gallun E, Bonnel A.M. Enhanced pitch sensitivity in individuals with autism: a signal detection analysis. J Cogn Neurosci 2003; 15: 226-35.

52. Kraus N, Micco A, Koch D et al. The mismatch negativity cortical evoked potential elicited by speech in cochlear-implant users. Hear Res 1993; 65: 118-24.

53. Roman S, Canévet G, Marquis P, Triglia J.M, LiÉGEOIS-ChAuvel C. Relationship between auditory perception skills and mismatch negativity recorded in free field in cochlearimplant users. Hear Res 2005; 201: 10-20.

54. Watson DR, Titterington J, Henry A, Toner JG. Auditory sensory memory and working memory processes in children with normal hearing and cochlear implants. Audiol Neurootol 2007; 12(2): 65-76.

55. Groenen P, Snik A, van der Broek P. On the clinical relevance of mismatch negativity: results from subjects with normal hearing and cochlear implant users. Audiol Neuro-Ool 1996; 1: 11224.

56. Kelly AS, Purdy SC, Thorne PR. Electrophysiological and speech perception measures of auditory processing in experienced adult cochlear implant users. Cin Neurophysiol 2005; 116: 1235-46.

57. Fischer C, Luaute J, Nemoz C, Morlet D, Kirkorian G, Mauguiere F. Improved prediction of awakening or nonawakening from severe anoxic coma using tree-based classification analysis. Orit Care Med 2006; 34: 1-5.

58. WiJnen VJM, van Boxtel GJM, Ellander HJ, de GeLDeR B. Mismatch negativity predicts recovery from the vegetative state. Cin Neurophysiol 2007; 118: 597-605.

59. Daltrozzo J, Wioland N, Mutschler V, Kotchoubey B. Predicting coma and other low responsive patients outcome using event-related brain potentials: a meta-analysis. Cin Neurophysiol 2007; 118: 606-14.

60. Kane N.M, Curry S.H, Butler S.R, Cummins B.H. Electrophysiological indicator of awakening from coma. Lancet 1993; 341: 688.

61. Kane N.M, Curry S.H, Rowlands C.A. et al. Event-related potentials neurophysiological tools for predicting emergence and early outcome from traumatic coma. Intensive Care Med 1996; 22(1): 39-46.

62. PeKKonen E. Mismatch Negativity in Aging and in Alzheimer's and Parkinson's Diseases. Audiol Neurootol 2000; 5: 216-24.

63. Todd J, Michie P, Schall U, Karayanidis F, Yabe H, NaAtanen R. Deviant Matters: Duration, Frequency, and intensity deviants reveal different patterns of mismatch negativity reduction in early and late schizophrenia. Biol Psychiatry 2008; 63(1): 58-64.

64. Michie PT, Budd TW, Todd J et Al. Duration and frequency mismatch negativity in schizophrenia. Cin Neurophysiol 2000; 111: 1054-65. 